\title{
High-temperature differential emission measure and altitude variations in the temperature and density of solar flare coronal $\mathrm{X}$-ray sources
}

\author{
Natasha L. S. Jeffrey ${ }^{1}$, Eduard P. Kontar ${ }^{1}$, and Brian R. Dennis ${ }^{2}$ \\ 1 School of Physics \& Astronomy, University of Glasgow, Glasgow G12 8QQ, UK \\ e-mail: natasha.jeffrey@glasgow.ac.uk \\ 2 Solar Physics Laboratory, Code 671, Heliophysics Science Division, NASA Goddard Space Flight Center, Greenbelt, \\ MD 20771, USA
}

Received 3 June 2015 / Accepted 13 October 2015

\begin{abstract}
The detailed knowledge of plasma heating and acceleration region properties presents a major observational challenge in solar flare physics. Using the Ramaty High Energy Solar Spectroscopic Imager (RHESSI), the high temperature differential emission measure, $\operatorname{DEM}(T)$, and the energy-dependent spatial structure of solar flare coronal sources were studied quantitatively. The altitude of the coronal X-ray source was observed to increase with energy by $\sim+0.2 \operatorname{arcsec} / \mathrm{keV}$ between 10 and $25 \mathrm{keV}$. Although an isothermal model can fit the thermal X-ray spectrum observed by RHESSI, such a model cannot account for the changes in altitude, and multithermal coronal sources are required where the temperature increases with altitude. For the first time, we show how RHESSI imaging information can be used to constrain the $\operatorname{DEM}(T)$ of a flaring plasma. We developed a thermal bremsstrahlung X-ray emission model with inhomogeneous temperature and density distributions to simultaneously reproduce i) $\operatorname{DEM}(T)$; ii) altitude as a function of energy; and iii) vertical extent of the flaring coronal source versus energy. We find that the temperature-altitude gradient in the region is $\sim+0.08 \mathrm{keV} / \operatorname{arcsec}(\sim 1.3 \mathrm{MK} / \mathrm{Mm})$. Similar altitude-energy trends in other flares suggest that the majority of coronal X-ray sources are multi-thermal and have strong vertical temperature and density gradients with a broad $\operatorname{DEM}(T)$.
\end{abstract}

Key words. Sun: flares - Sun: corona - Sun: X-rays, gamma rays - Sun: particle emission

\section{Introduction}

Over the past decade, the X-ray imaging spectroscopy of the Ramaty High Energy Solar Spectroscopic Imager (RHESSI; Lin et al. 2002) has allowed changes in the spatial properties of solar flare X-ray sources to be studied in detail. Along with high energy resolution X-ray spectroscopy $(\leq 1 \mathrm{keV}$ at $3 \mathrm{keV}$ increasing to $5 \mathrm{keV}$ at $5 \mathrm{MeV}$ ), RHESSI is capable of indirect X-ray imaging using nine rotating modulation collimators (RMCs) giving angular resolutions between 2". 3 and 183". In practice, the angular resolution is usually $\gtrsim 5^{\prime \prime}$ (full width half maximum - FWHM) due to finite counting statistics and image reconstruction uncertainties. However, RHESSI is capable of inferring changes in the positions of X-ray sources down to the sub-arcsecond level. Sub-arcsecond measurements of X-ray footpoint locations have been achieved using X-ray visibilities (see e.g. Kontar et al. 2008, 2010; Jeffrey \& Kontar 2013), improving upon forward fitting a Gaussian source model to the RHESSI modulated lightcurves (e.g. Aschwanden et al. 2002).

Recently, many studies have investigated flares where the majority of X-ray emission comes from a coronal thick-target source (e.g. Veronig \& Brown 2004), in contrast to the usual flare that is dominated by footpoint hard X-ray (HXR) emission. The first energy dependent spatial study of such coronal X-ray sources (Xu et al. 2008) and further works (e.g. Guo et al. 2012 , 2013) examined how X-ray source lengths (the direction that appears to lie parallel to a guiding magnetic field) changed with X-ray energy. Such observations, with the help of numerical simulation (Jeffrey et al. 2014), have allowed the estimation of coronal plasma number density and the acceleration rate of electrons within the region. Kontar et al. (2011b) found increasing X-ray source widths (defined in the direction perpendicular to the guiding field) with energy, a trend consistent with magnetic turbulence in the flaring coronal source. Jeffrey \& Kontar (2013) studied the temporal evolution of radial positions (altitudes), lengths and widths of such coronal X-ray sources.

Changes in coronal X-ray source radial position (or altitude) with X-ray energy have not been studied extensively in the RHESSI era. Unlike the changes in X-ray source size, an increasing altitude with time is often observed for all types of flaring coronal X-ray sources, and the trend is often seen in other wavelengths such as UV, extreme-UV (EUV) and soft X-rays (e.g. Forbes \& Acton 1996). In a standard flare model, the upward motion of coronal X-ray sources with time is often explained by the upward motion of a magnetic reconnection site, with loops reconnecting continuously at increasing altitude and then cooling (e.g. Svestka et al. 1987; Tsuneta et al. 1992; Švestka 1996; Gallagher et al. 2002). Sometimes an initial decrease in altitude is followed by an increase in altitude after the impulsive phase of the flare (Sui \& Holman 2003; Sui et al. 2004; Veronig et al. 2006; Liu et al. 2009; Joshi et al. 2009; Reznikova et al. 2010; Gosain 2012; Jeffrey \& Kontar 2013), and sometimes even more complicated motions are observed (e.g. Liu et al. 2013). These observations are viewed as an argument to support the standard flare scenario involving magnetic reconnection above the coronal source. Despite RHESSI's unprecedented spectral resolution, the temperature structure (differential 


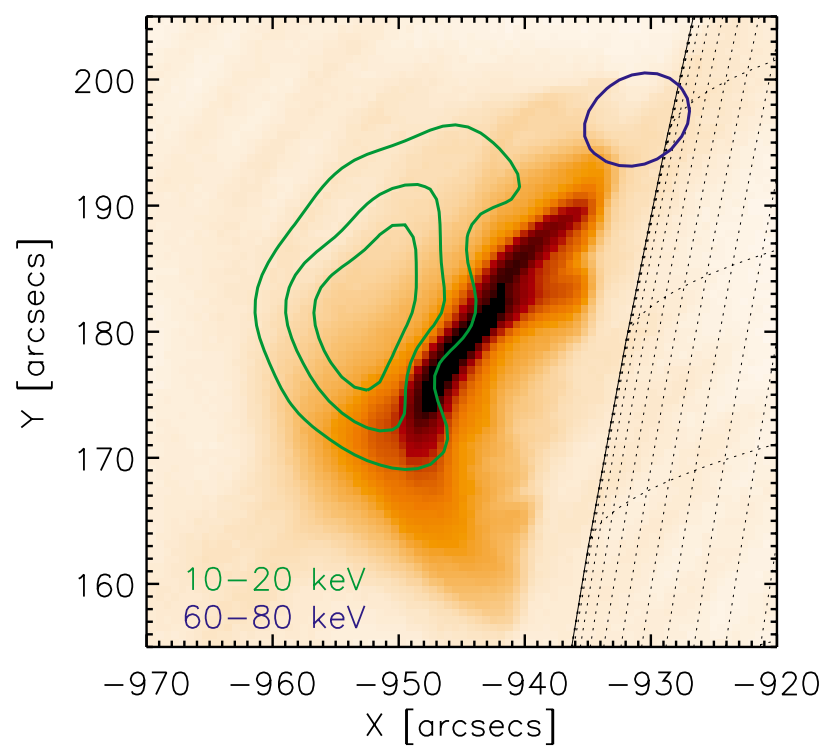

Fig. 1. SDO AIA $94 \AA$ image at 02:08:40 UT (red-orange background). RHESSI CLEAN contours at the time interval of 02:08 to 02:10 UT for $10-20 \mathrm{keV}$ (green) at 30, 50 and $70 \%$ of the maximum, and for $60-80 \mathrm{keV}$ (blue) at the $50 \%$ level.

emission measure $\operatorname{DEM}(T))$ above $T \sim 1 \mathrm{keV}$ remains poorly determined (e.g. Prato et al. 2006).

In this paper, we present a spatial-spectral study of flaring coronal X-ray sources. A limb flare SOL2013-05-13T02:12 is studied in detail (the time of 02:12 UT is the RHESSI peak flux time). We deduce the changes in coronal X-ray source location with energy at a given time and find a relationship between X-ray source energy and altitude. During this study, we are not concerned with the physical cause of the trend, only how RHESSI imaging information can be used to further constrain the $\operatorname{DEM}(T)$, so that it is consistent with both RHESSI spectral and imaging observations. Further, an isothermal model, often used to fit the X-ray spectrum at low energies, is shown to be inconsistent with RHESSI imaging observations for the flares studied.

\section{The observation of SOL2013-05-13T02:12}

GOES X1.7 flare SOL2013-05-13T02:12 was chosen for detailed analysis because it had a strong coronal X-ray source located at the eastern limb. A limb flare was chosen so that the radial distance changes correspond to height changes with minimal projection effects. This flare has one visible northern footpoint that can be seen up to energies of $\sim 100 \mathrm{keV}$. Figure 1 shows a loop filled with hot plasma imaged with the Solar Dynamics Observatory Atmospheric Imaging Assembly (SDO AIA; Lemen et al. 2012) in the $94 \AA$ passband. RHESSI $10-20 \mathrm{keV}$ and $60-80 \mathrm{keV}$ contours are displayed. The $10-20 \mathrm{keV}$ emission comes from the flare coronal source located above the $94 \AA$ loop, while the bulk of the $60-80 \mathrm{keV}$ emission comes from a northern footpoint source located close to the footpoint of the $94 \AA$ loop. The RHESSI and GOES lightcurves for the flare are shown in Fig. 2. There is a gradual rise in the X-ray flux up to $50 \mathrm{keV}$ from 01:56 UT onwards, with sharper increases in the 50-100 keV and 100-300 keV bands between 01:56 and 02:30 UT.
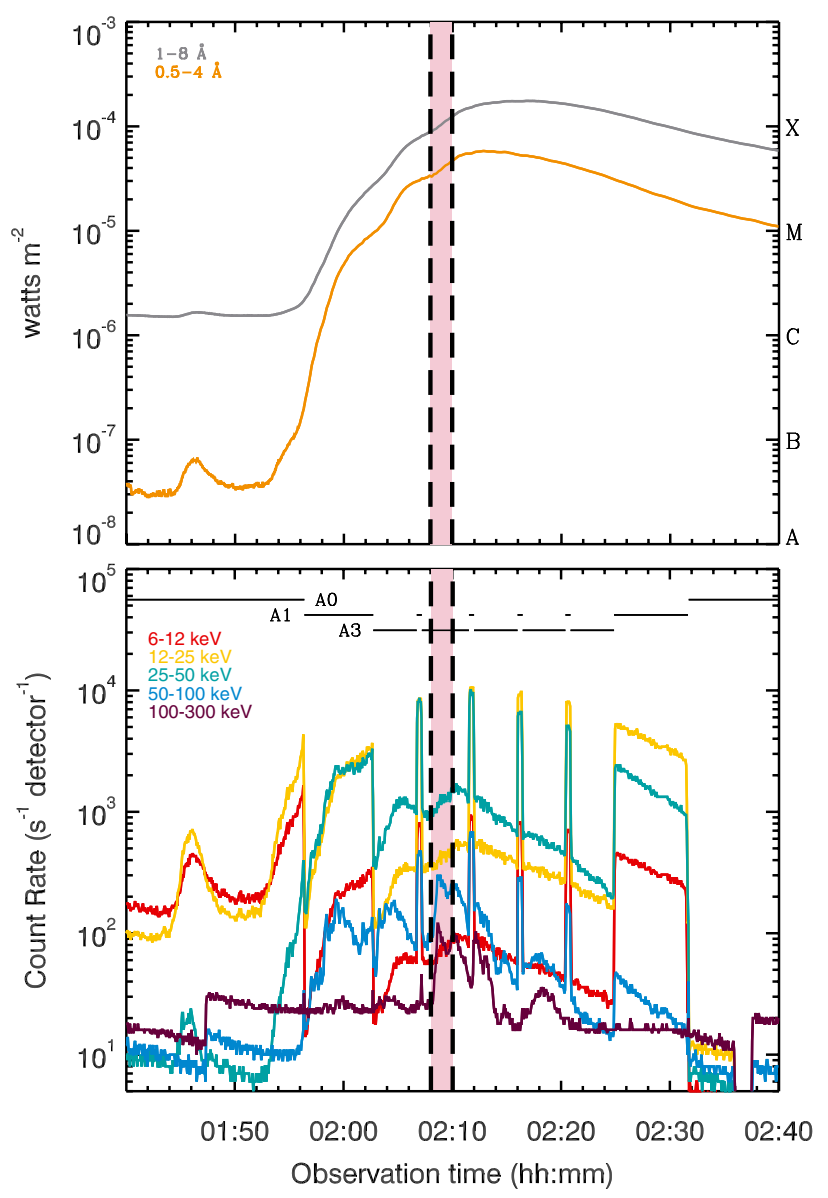

Fig. 2. GOES (top: $1-8 \AA$ and $0.5-4 \AA$ ) and RHESSI (bottom: 6-12, 12-25, 25-50, 50-100 and 100-300 keV) lightcurves for the chosen flare SOL2013-05-13T02:12. The two minute time of study from 02:08 to $02: 10$ UT is shown by the pink band between the two vertical dashed lines. The "jumps" in the RHESSI lightcurve occur due to instrumental attenuation changes reducing the X-ray flux reaching the detectors. At this time, RHESSI was in attenuator state A3, meaning that both the thin and thick attenuators cover the detectors.

\section{RHESSI spectroscopy and imaging}

A spectral analysis of the flare was performed for the time interval from 02:08 to 02:10 UT. The count rate for this X-class flare is high so spectroscopy using a single detector could be performed. Comparison of individual spectra from detectors 1 to 9 showed that detector 6 had the best energy resolution, and shows the spectral features between 6 to $10 \mathrm{keV}$. As expected, the spectrum during this time interval has a strong thermal component below $30 \mathrm{keV}$ and a power-law spectrum at higher energies up to $\sim 150 \mathrm{keV}$ (see Fig. 3). Since RHESSI is in attenuator state A3 (the thin and thick attenuators cover the detectors), the lower energy limit was set to $6 \mathrm{keV}$. The majority of the counts recorded below $6 \mathrm{keV}$ are not incident photons at that energy; they are from high energy photons producing K-shell escape photons from the germanium detector itself (see e.g. Phillips et al. 2006).

Using the Object Spectral Executive (OSPEX software, Schwartz et al. 2002), the following five functions describing the thermal component were fitted to the background-subtracted count fluxes in the energy range between 6 and $100 \mathrm{keV}$ :

1. The isothermal function (f_vth), "Fit 1", provides the temperature $T[\mathrm{keV}]$ and emission measure $E M\left[\mathrm{~cm}^{-3}\right]$ of the 
Table 1. A parameter comparison of the thermal fitting functions shown in Fig. 3.

\begin{tabular}{|c|c|c|c|c|c|c|c|c|}
\hline Fit function & $\begin{array}{c}T_{1} \\
{[\mathrm{keV}]}\end{array}$ & $\begin{array}{c}T_{2} \\
{[\mathrm{keV}]}\end{array}$ & $\begin{array}{c}T_{\text {peak }} \\
{[\mathrm{keV}]}\end{array}$ & $\begin{array}{c}E M_{1} \times 10^{49} \\
{\left[\mathrm{~cm}^{-3}\right]}\end{array}$ & $\begin{array}{c}E M_{2} \times 10^{49} \\
{\left[\mathrm{~cm}^{-3}\right]}\end{array}$ & $\begin{array}{c}\operatorname{DEM}(2 \mathrm{keV}) \times 10^{49} \\
{\left[\mathrm{keV}^{-1} \mathrm{~cm}^{-3}\right]}\end{array}$ & $\begin{array}{c}\operatorname{DEM}\left(T_{\text {peak }}\right) \times 10^{49} \\
{\left[\mathrm{keV}^{-1} \mathrm{~cm}^{-3}\right]}\end{array}$ & $\chi^{2}$ \\
\hline vth & 2.5 & $\mathrm{X}$ & $\mathrm{X}$ & 0.9 & $\mathrm{X}$ & $\mathrm{X}$ & $\mathrm{X}$ & 1.3 \\
\hline $2 \mathrm{vth}$ & 1.7 & 2.7 & $\mathrm{X}$ & 3.4 & 0.4 & $\mathrm{X}$ & $\mathrm{X}$ & 1.0 \\
\hline multi_therm_pow & $\mathrm{X}$ & $X$ & $\mathrm{X}$ & $\mathrm{X}$ & $\mathrm{X}$ & 2.0 & $\mathrm{X}$ & 1.0 \\
\hline multi_therm_gauss & $\mathrm{X}$ & $\mathrm{X}$ & 1.4 & $\mathrm{X}$ & $\mathrm{X}$ & $\mathrm{X}$ & 5.7 & 1.0 \\
\hline multi_therm_pow_exp & $X$ & $\mathrm{X}$ & 1.4 & 5.2 & $\mathrm{X}$ & $\mathrm{X}$ & $\mathrm{X}$ & 1.0 \\
\hline
\end{tabular}

thermal source. The emission measure and temperature are free parameters while the relative iron abundance is fixed at 1.0 times the CHIANTI atomic database (Dere et al. 1997; Landi et al. 2013) coronal value, which is the default OSPEX value (investigating different relative iron abundances is beyond the scope of the paper). The relative iron abundance is fixed at this value for all fits (1) to (5) (see Table 1 and Fig. 3 for all parameters and fits).

2. The double isothermal function (f_2vth), "Fit 2", is the sum of two isothermal functions each with their own emission measures $\left(E M_{1}, E M_{2}\right)$ and temperatures $\left(T_{1}, T_{2}\right)$, but with the same, fixed relative iron abundance. $E M_{1}, E M_{2}, T_{1}, T_{2}$ are all free parameters.

3. A multi-thermal power-law function (f_multi_therm_pow), "Fit 3", relating the differential emission measure $\operatorname{DEM}(T)$ $\left[\mathrm{cm}^{-3} \mathrm{keV}^{-1}\right]$ to the temperature $T$ by,

$$
\operatorname{DEM}(T)=\operatorname{DEM}(T=2 \mathrm{keV})\left(\frac{2}{T}\right)^{\beta}
$$

where $\beta$ is the power-law index and $\operatorname{DEM}(2 \mathrm{keV})$ is the differential emission measure at a temperature of $2 \mathrm{keV}$. The function also provides a minimum and maximum value of temperature $T$. The relative iron abundance is fixed, while all other parameters are free.

4. A multi-thermal Gaussian in $\log _{10} T$ (f_multi_therm_gauss), "Fit 4", relates the DEM to the temperature $T$ using,

$$
\operatorname{DEM}(T)=\operatorname{DEM}\left(T_{\text {peak }}\right) \exp \left(-\frac{\left(\log _{10} T-\log _{10} T_{\text {peak }}\right)^{2}}{2 \sigma^{2}}\right)
$$

where the DEM is a Gaussian in logarithmic temperature space, $T_{\text {peak }}[\mathrm{keV}]$ is the temperature at the peak, $\operatorname{DEM}\left(T_{\text {peak }}\right)$ is the DEM at $T_{\text {peak }}$ and $\sigma$ is the standard deviation of the Gaussian in units of $\log _{10} \mathrm{keV}$. The relative iron abundance, and the minimum and maximum temperatures, are fixed. $\operatorname{DEM}\left(T_{\text {peak }}\right), T_{\text {peak }}$ and $\sigma$ are free parameters.

5. Finally a multi-thermal power-law and exponential function (f_multi_therm_pow_exp), "Fit 5" relates the DEM to the temperature using,

$$
\operatorname{DEM}(T)=\frac{E M}{\zeta T_{\text {peak }} \Gamma(\zeta-1)}\left(\frac{\zeta T_{\text {peak }}}{T}\right)^{\zeta} \exp \left(-\frac{\zeta T_{\text {peak }}}{T}\right)
$$

where $\Gamma(x)$ is the gamma function, $E M\left[\mathrm{~cm}^{-3}\right]$ is the total emission measure integrated from the minimum to the maximum temperature, $T_{\text {peak }}$ is the peak temperature of the DEM and $\zeta$ is the power-law index. $E M, T_{\text {peak }}$ and $\zeta$ are free parameters while the relative iron abundance, and minimum and maximum temperatures, are fixed.
The following functions were also included in each case (see Fig. 3): thick-target bremsstrahlung (f_thick2_vnorm) to account for non-thermal emission from a power-law distribution of electrons above $\sim 30 \mathrm{keV}$, a pileup correction ${ }^{1}$, a function to fine tune the RHESSI detector response matrix (f_drm_mod), and line components (f_line) to account for instrumental features near $\sim 10 \mathrm{keV}$.

Our spectral fit results show that it is impossible to constrain the overall shape of the $\operatorname{DEM}(T)$ below $\sim T=1.5 \mathrm{keV}$ with RHESSI data. The isothermal function (which is commonly used in RHESSI spectral analysis, see e.g. Holman et al. 2011; Kontar et al. 2011a, as reviews) is a marginally worse fit in terms of the reduced $\chi^{2}$ and the residuals below $15 \mathrm{keV}$ (Table 1 and Fig. 3). Otherwise, all thermal models can adequately fit the thermal part of the spectrum. This result clearly demonstrates that a wide range of temperature distributions (summarised in Fig. 4) with various $\operatorname{DEM}(T)$ are consistent with the measured RHESSI count flux spectrum. In Fig. 4, the DEM for Fit 3 (grey), Fit 4 (orange) and Fit 5 (blue) are shown. Over the temperature range of $\sim 1.6 \mathrm{keV}$ to $3.0 \mathrm{keV}$, the OSPEX model DEMs agree within the model errors (shaded areas), suggesting that RHESSI can constrain the form of the $\operatorname{DEM}(T)$ between this temperature range, regardless of the chosen DEM model. The shaded areas are derived from the formal uncertainties on each free parameter in the models determined assuming Poisson statistics are the only source of error and that all are orthogonal. A more detailed Monte Carlo analysis would be needed to determine these uncertainties more accurately using the methods described in Ireland et al. (2013), but the range of applicability of each model shown in Fig. 4 is adequate for our current purposes.

\subsection{RHESSI imaging at various energies in the thermal range}

The flare was imaged using RHESSI detectors 3-7 over five energy bands (10-11, 11-13, 13-16, 16-20 and 20-25 keV), where the coronal X-ray emission dominated (Fig. 1). Detectors $1,2,8$ and 9 were not used. Detector 1 showed no modulation (the source was over-resolved with significant noise), detector 2 is only sensitive above $\sim 20 \mathrm{keV}$, and the resolutions of detectors 8 and $9\left(106^{\prime \prime}\right.$ and $183^{\prime \prime}$ respectively) are larger than the image area of $64^{\prime \prime} \times 64^{\prime \prime}$. The images were created using two imaging algorithms: CLEAN (Högbom 1974; Hurford et al. 2002) and Visibility Forward Fitting (Vis_FwdFit; Schmahl et al. 2007) using a 1 arcsecond pixel size. CLEAN images show a loop-like structure visible up to the 20-25 keV bin. Such a loop-like structure is well suited for fitting a simple curved

1 (f_pileup_mod) The pile-up component accounts for those photons arriving at the detector at nearly the same time, that are detected as a single count with an energy equal to the sum of the individual photon energies. 

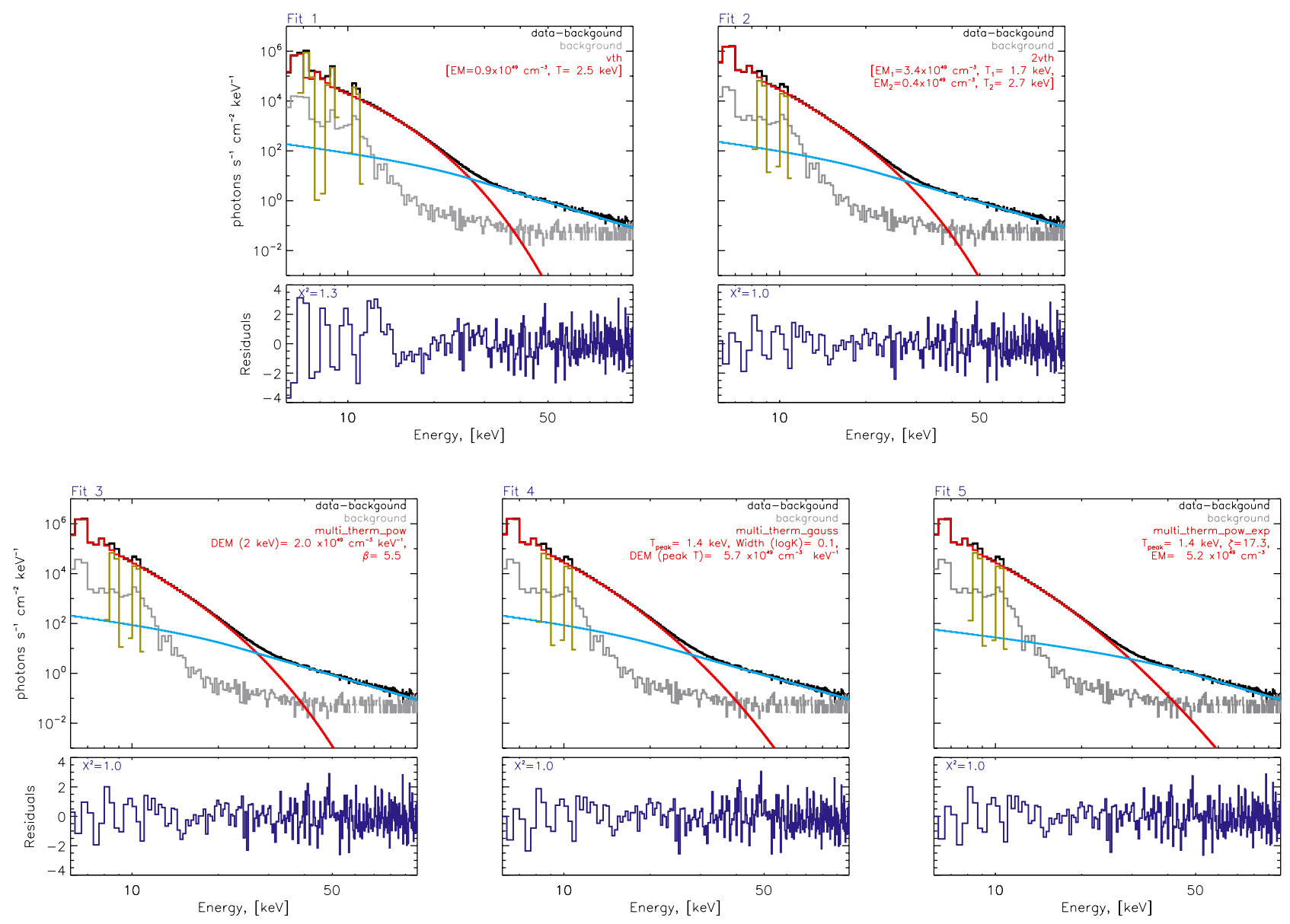

Fig. 3. Photon flux spectrum fitted with different functions describing the thermal component. Top left: single isothermal, Top right: double isothermal, Bottom left: multi-thermal power law. Bottom middle: multi-thermal Gaussian in $\log _{10} T$ and bottom right: multi-thermal power-law and exponential. The normalised residuals are plotted below each spectrum, and each is created using only detector 6 and the functions fit the count spectrum between 6 and $100 \mathrm{keV}$ for a chosen time interval of 02:08 to 02:10 UT. The values of all thermal parameters are shown in the figure and in Table 1. The gold lines in each panel represent Gaussian line fits (line) compensating for instrumental anomalies in the RHESSI spectrum. A thick2_vnorm function (light blue) is used to account for the non-thermal X-ray emission at higher energies.

elliptical Gaussian fit, using Vis_FwdFit ${ }^{2}$, to RHESSI's X-ray visibilities, so that the location and the characteristic sizes of the X-ray source can be found. The Vis_FwdFit $\chi^{2}$ values determine whether the parameters provided by the fit are acceptable. We note that Vis_FwdFit actually provides the fitted curve centre of mass and this is not equivalent to the loop top position which is required for the study. Instead, the image peak is found using the SolarSoftWare (SSW) routine parapeak.pro. This program estimates the peak position by performing a parabolic fit to the image data. An error is found using the standard deviation of the parabolic fit. The CLEAN image peak is also found using the same method so that the positions provided by each algorithm can be compared. Figure 5 shows a CLEAN image for an energy range of 13-16 keV. Vis_FwdFit 50\% contours at $10-11 \mathrm{keV}, 13-16 \mathrm{keV}$ and $20-25 \mathrm{keV}$ are displayed. Figure 5 also shows a footpoint source at $60-80 \mathrm{keV}$.

\subsection{The relationship between $X$-ray energy and radial distance}

Using the CLEAN and Vis_FwdFit image peak positions $(x, y)$ provided by parapeak.pro, we calculated the radial distances

\footnotetext{
2 http://hesperia.gsfc.nasa.gov/rhessi3/software/ imaging-software/vis-fwdfit/index.html
}

$R=\sqrt{x^{2}+y^{2}}$ (measured from the solar disk centre) for each algorithm at each energy range. An error for $R$ is found from error propagation. Figure 5 (right) shows that the calculated $R$ errors are small for this flare (approximately half an arcsecond or less) at the chosen energy ranges and time, and that the data from two imaging algorithms give a similar trend - the higher the energy, the higher the location of the peak. Both results can be well-fitted by a straight line, so that the observed relationship between radial distance $R$ and energy $\epsilon$ is approximated by

$\frac{\mathrm{d} R(\epsilon)}{\mathrm{d} \epsilon}=\alpha, \quad\left[\operatorname{arcsec} \mathrm{keV}^{-1}\right]$

where $\alpha$ is a constant gradient. Both algorithms give a similar gradient $\mathrm{d} R / \mathrm{d} \epsilon$ equal to $0.24 \pm 0.02 \operatorname{arcsec} / \mathrm{keV}$ for Vis_FwdFit and $0.20 \pm 0.01 \mathrm{arcsec} / \mathrm{keV}$ for CLEAN, which is important for the analysis. Comparing both algorithms, the absolute values of $R(\epsilon)$ are shifted by $\pm 0.3-0 !$, with the CLEAN values at a lower height than the Vis_FwdFit values. This is due to differences in the way each algorithm reconstructs an image. The CLEAN algorithm creates an image by finding point sources and convolving them with the instrumental point spread function (PSF). The image peak simply corresponds to the brightest point smoothed out by the PSF. Since Vis_FwdFit fits a chosen distribution such as a Gaussian to the X-ray visibilities, the peak of a given source is determined by the fitted distribution to 
N. L. S. Jeffrey et al.: The temperature and density structures of coronal X-ray sources

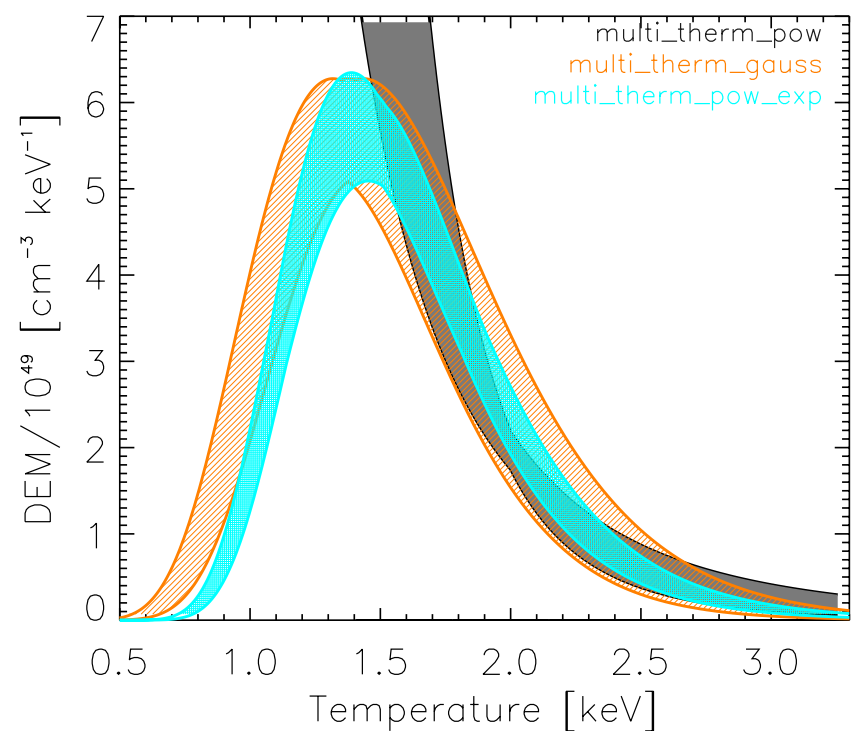

Fig. 4. Differential emission measures, $\operatorname{DEM}(T)$, obtained for the various multi-thermal fits (Fig. 3). The shaded areas indicate the estimated confidence intervals. The form of all $\operatorname{DEM}(T) \mathrm{s}$ are similar between $\sim 1.6$ and $3.0 \mathrm{keV}$, independent of the chosen model $\operatorname{DEM}(T)$.

the data. Hence, we should not expect both algorithms to produce the X-ray source peaks in exactly the same locations. For the purposes of the study, Vis_FwdFit is a more suitable algorithm. However, the systematic difference between both algorithms is very small (sub-arcsecond), regardless of the fact they both create the image in a completely different way. Basically, the CLEAN algorithm is used to provide additional confidence in the results. Other factors, such as the RHESSI PSF should not alter the locations of the centroids at different energies. The only variable with energy is the transmission of the material overlying the detectors - the attenuators, thermal blankets, and cryostat cover. While the overall absolute X-ray attenuation of this material is not so well known, its variation with energy is very well known and hence should not produce any systematic effect of the altitude estimates.

\subsection{The vertical extent of the $X$-ray sources at each energy}

Following Jeffrey \& Kontar (2013), we determine the characteristic sizes of the coronal sources, using Vis_FwdFit. The loop FWHM, $W$, of each X-ray source is found for every energy band (Fig. 6). The loop width is defined as the X-ray source FWHM in the radial direction, measured at the loop top, perpendicular to the axis of the curved elliptical Gaussian. As shown in Fig. 6, at energies of 10 to $25 \mathrm{keV}$, the X-ray source width $W$ lies between $\sim 10$.'5 and $12^{\prime \prime}$ and appears to decrease with increasing energy. The mean X-ray source width is $\langle W\rangle=11^{\prime \prime} .2 \pm 0$. . 5 . $W$ is also found from Vis_FwdFit using three wider energy bins of $10-12.5 \mathrm{keV}, 12.5-18 \mathrm{keV}$ and $18-25 \mathrm{keV}$ to increase the number of counts per band, with $W$ appearing to remain constant with energy, at least within the errors. This gives an average width $\langle W\rangle=11^{\prime \prime} .0 \pm 0$.' 3 . We note that the width of $\sim 11^{\prime \prime}$ of each X-ray source is much larger than the observed energy dependent shift in altitude of only $\sim 3^{\prime \prime}$.

\section{The peak of X-ray emission and $\operatorname{DEM}(T)$}

From RHESSI observations (see Fig. 5), we found that the flare coronal X-ray source altitude increases with energy, according to the approximate linear relation (4). Hence

$\frac{\mathrm{d} z}{\mathrm{~d} \epsilon}=\alpha$

where $z$ is the height above the solar limb.

Our task is to find an analytical model that can produce the observed $z$ versus $\epsilon$ trend and relate such changes to a temperature $T(z)$ structure and a number density $n(z)$ structure in the flaring coronal region. To do this, we derive a relationship between the altitude $z$ of the X-ray source and energy $\epsilon$.

Since the spectroscopy results for the flare show that the spectrum in the range $\sim 6$ to $25 \mathrm{keV}$ can be well-fitted by multi-thermal models (Fig. 3), we assume that all the coronal source X-rays in this range are emitted as multi-thermal bremsstrahlung. This assumption is supported by the fact that the thermal emission dominates the non-thermal emission in this range. The spectroscopy results (Fig. 3) show that the thermal and non-thermal components are equal at $\sim 28 \mathrm{keV}$ with the thermal component being increasingly dominant at lower energies. Consequently, we assume that all the emission in the 6 to $25 \mathrm{keV}$ energy range is multi-thermal bremsstrahlung from the flaring coronal source.

Consider an emitting volume $\mathrm{d} V=A \mathrm{~d} z$, where $A$ is the X-ray source area perpendicular to the radial direction (altitude) and image plane, as shown in Fig. 7. The photon flux emitted as thermal bremsstrahlung per unit energy $\epsilon$ per unit height $z$ from a plasma characterised by a temperature $T[\mathrm{keV}]$ and number density $n\left[\mathrm{~cm}^{-3}\right]$ is given by e.g. Tandberg-Hanssen \& Emslie (1988)

$J(\epsilon, z) \propto \frac{n^{2}(z) A(z)}{\epsilon \sqrt{T(z)}} \exp \left(-\frac{\epsilon}{T(z)}\right)$

where $J(\epsilon, z)$ is the photon flux per unit of height [photons $\mathrm{s}^{-1} \mathrm{~cm}^{-2} \mathrm{keV}^{-1} \mathrm{~cm}^{-1}$ ].

The total photon flux [photons s${ }^{-1} \mathrm{~cm}^{-2} \mathrm{keV}^{-1}$ ] integrated over all $z$ is then given by,

$I(\epsilon)=\int_{0}^{\infty} J(\epsilon, z) \mathrm{d} z$

At any observed energy $\epsilon, \mathrm{X}$-ray flux $J(\epsilon, z)$ should have maximum at $z(\epsilon)$ determined by the derivative

$\frac{\partial J}{\partial z} \propto \frac{\partial}{\partial z}\left[\frac{n^{2} A}{\epsilon \sqrt{T}} \exp \left(-\frac{\epsilon}{T}\right)\right]=0$.

Re-arranging Eq. (8) for $\epsilon(z)$ one finds,

$\epsilon(z)=\frac{T(z)}{2}-\frac{\mathrm{d}\left(n^{2} A\right)}{\mathrm{d} z} \frac{\mathrm{d} z}{\mathrm{~d} T} \frac{T^{2}}{n^{2} A}$

and we can write:

$\epsilon(z)=\frac{T(z)}{2}-\frac{\mathrm{d}\left(n^{2} A\right)}{\mathrm{d} T} \frac{T^{2}}{n^{2} A}$.

The plasma density $n(z)$ and temperature $T(z)$ also determine the differential emission measure

$\operatorname{DEM}(T)=n^{2} \frac{\mathrm{d} V}{\mathrm{~d} T}$.

Using the definition of $\operatorname{DEM}(T)$, one can write

$\operatorname{DEM}(T)=\left.n^{2} \frac{\mathrm{d} V}{\mathrm{~d} T}\right|_{z=z(T)}=\left.n^{2} A \frac{\mathrm{d} z}{\mathrm{~d} T}\right|_{z=z(T)}$

where $z=z(T)$ is the height at a temperature $T$.

$\operatorname{Both} \operatorname{DEM}(T)$ and the peak position of the X-ray flux at a given energy $\epsilon(z)$ are uniquely determined by $T(z)$ and $n^{2}(z) A(z)$. 

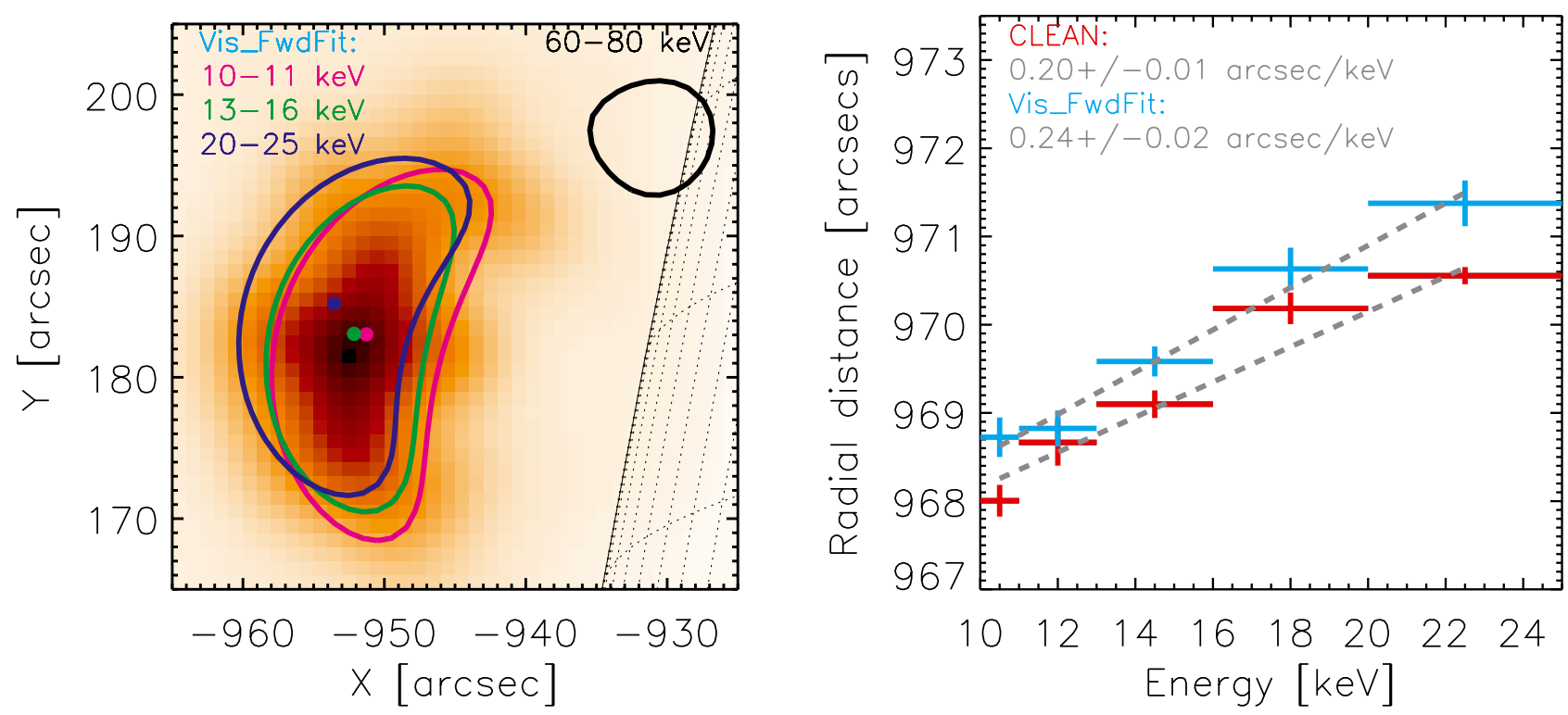

Fig. 5. Left: CLEAN image for a time interval from 02:08 to $02: 10$ UT at 13-16 keV with a CLEAN 60-80 keV 50\% contour (black). Vis_FwdFit contours at 50\% of the maximum and coronal source positions (coloured dots) are displayed for three energy ranges (10-11 keV (pink), 13-16 keV (green) and 20-25 keV (blue)) showing the increasing altitude of the coronal source location with energy. Right: the peak radial position $R$ plotted against X-ray energy $\epsilon$, for both the CLEAN (red) and Vis_FwdFit (blue) algorithms. The gradient $\alpha=\mathrm{d} R / \mathrm{d} \epsilon \pm \mathrm{error}$ found from each linear fit is shown (grey dashed lines) on the graph.

\subsection{Isothermal plasma}

Let us first assume a simple case. For an isothermal plasma $\mathrm{d} T / \mathrm{d} z=0$ and hence $\mathrm{d}\left(n^{2} A\right) / \mathrm{d} T=0$, Eq. (10) becomes

$\epsilon_{0}=\frac{T}{2}=\frac{T_{0}}{2}$

over all $z$, where $T=T_{0}$ is a constant. Therefore, an isothermal plasma cannot account for the observed changes in peak X-ray energy with height $z$. Importantly, this shows that an isothermal plasma in the flaring coronal source is ruled out by imaging data, even though an isothermal bremsstrahlung function can reasonably fit the solar flare spectrum found using RHESSI (see Fig. 3).

\subsection{Height-dependent temperature with constant $n^{2} A$}

If $n^{2} A$ is constant with height $z$, i.e. $\mathrm{d}\left(n^{2} A\right) / \mathrm{d} z=0$, then Eq. (9) yields

$\epsilon_{0}=\frac{T(z)}{2}$.

Since the observed X-ray energies are 10 to $25 \mathrm{keV}$, the temperatures $T(z)$ in a constant $n^{2} A$ plasma would have to be of the order 20 to $50 \mathrm{keV}$, i.e. 230 to $580 \mathrm{MK}$. From the RHESSI spectrum, we see that the majority of the emitted thermal X-rays are emitted at lower energies corresponding to lower temperature sources of 1 to $3 \mathrm{keV}$, and hence a constant $n^{2} A$ is unlikely.

\subsection{Height-dependent $T(z)$ and $n^{2}(z) A(z)$}

In Eq. (10), if the gradient $\mathrm{d}\left(n^{2} A\right) / \mathrm{d} T \neq 0$, then $\mathrm{d}\left(n^{2} A\right) / \mathrm{d} T=$ $\left(\mathrm{d}\left(n^{2} A\right) / \mathrm{d} z\right)(\mathrm{d} z / \mathrm{d} T)$ must be negative for energy $\epsilon$ to be positive, or the second term must be smaller than the first term on the RHS of Eq. (10). Since we observe higher energy X-ray sources above lower energy X-ray sources, the temperature must increase with altitude, so that $\mathrm{d} T / \mathrm{d} z>0$. Hence, in this scenario $\mathrm{d}\left(n^{2} A\right) / \mathrm{d} z<0$ for $\mathrm{d}\left(n^{2} A\right) / \mathrm{d} T<0$. However, $\mathrm{d}\left(n^{2} A\right) / \mathrm{d} z$ does not have to be negative over all $z$, only over a portion of $z$ where we see the X-ray peak positions at a given energy $\epsilon$. Actually, if $\mathrm{d}\left(n^{2} A\right) / \mathrm{d} z$ is negative over the entire region, then the peak positions may appear at a lower altitude than suggested by a given observation, but this is further investigated via modelling. It is more difficult to determine the form of $\epsilon$ versus $z$ for the case where $\mathrm{d}\left(n^{2} A\right) / \mathrm{d} z$ is positive, and the second term is smaller than the first term. Hence modelling is required to further determine the forms of $T(z)$ and $n^{2}(z) A(z)$ that can account for both RHESSI imaging and spectroscopic observations.

\section{Modelling the vertical temperature and number density distributions}

Using X-ray imaging and spectroscopy data as a guide, we develop a model of $T(z)$ and $n^{2}(z) A(z)$, which can simultaneously explain flare X-ray imaging and spectroscopic observations.

Firstly, we note that the density $n(z)$ and the area perpendicular to the image plane $A(z)$ (see Fig. 7 for the geometry) appear in Eqs. (10) and (11) as a combination $n^{2}(z) A(z)$. Therefore, the observations cannot say anything further about the individual distributions of $n(z)$ and $A(z)$, only about the combination $n^{2}(z) A(z)$. However, we can deduce the form of $n^{2}(z) A(z)$ and the temperature structure $T(z)$ from the observations.

The modelled $n^{2}(z) A(z)$ and $T(z)$ should reproduce the following main characteristics of X-ray emission determined from RHESSI observations:

1. The observed X-ray source widths (using a Gaussian fit) should have a $F W H M \sim 11^{\prime \prime}$ with visible X-ray emission over $20^{\prime \prime}$ in the radial direction between the energies of 10 to $25 \mathrm{keV}$. The minimum visible X-ray emission should be at a height $\sim 10^{\prime \prime}$ above the limb.

2. Altitude $z$ versus peak energy $\epsilon$ should be well-fitted by Eq. (10), relating energy, height, $T(z)$ and $n^{2}(z) A(z)$. The 
N. L. S. Jeffrey et al.: The temperature and density structures of coronal X-ray sources
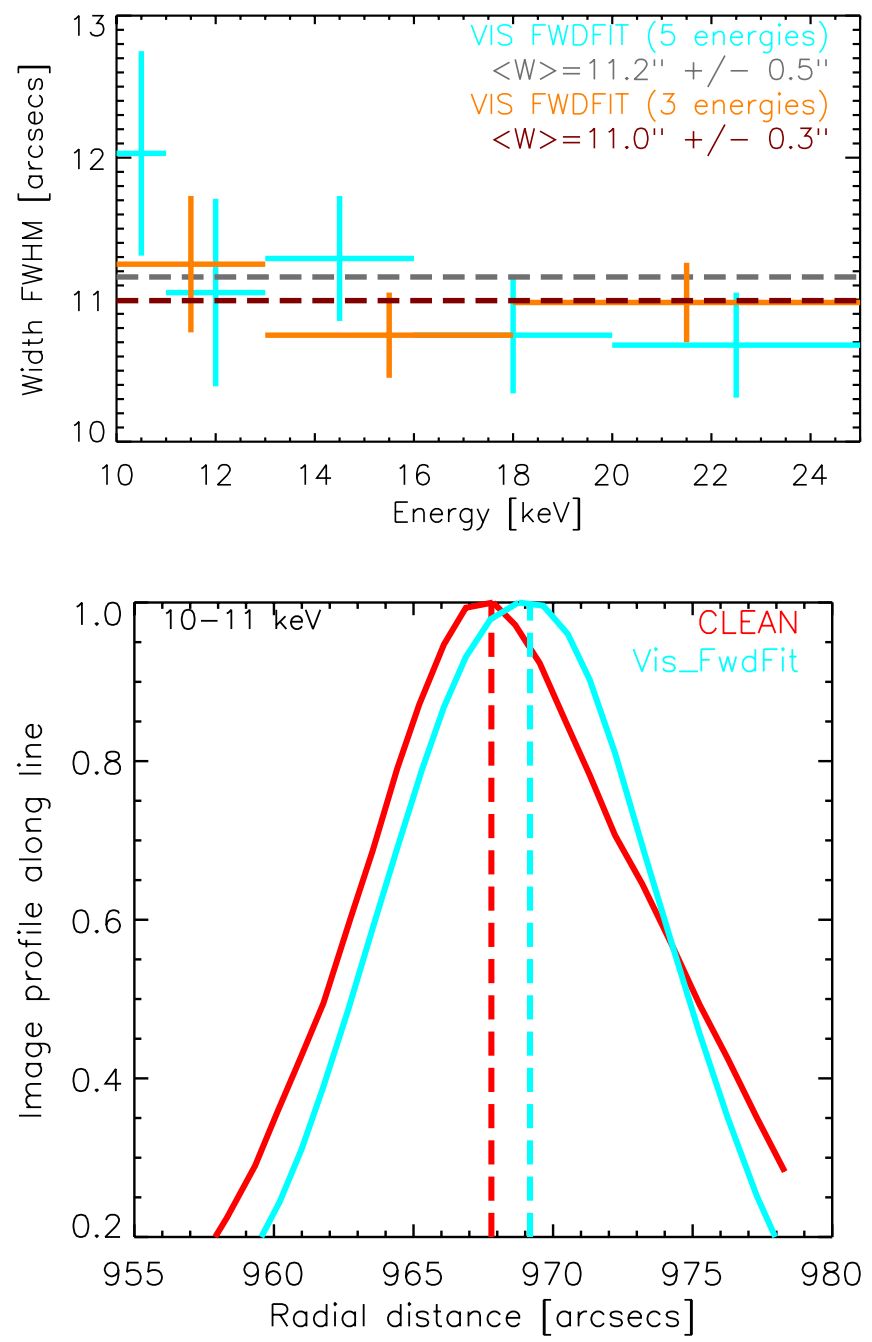

Fig. 6. Top: loop width FWHM $W$ found from Vis_FwdFit over the same energy ranges as for the $(x, y)$ peak positions shown in Fig. 5. The average width or vertical size of an X-ray source in the radial direction $R$ is $\langle W\rangle=11^{\prime \prime} 2 \pm 0$.'5. The width was also found using three larger energy bins of 10-12.5 keV, 12.5-18 keV and 18-25 keV, increasing the number of counts and hence reducing the uncertainty. This gives an average width of $\langle W\rangle=11^{\prime \prime} 0 \pm 0$. .' 3 . Bottom: CLEAN (red) and Vis_FwdFit (blue) image profiles along a line perpendicular to the solar surface and through the centroid position of the coronal source, for the energy bin of 10-11 keV. Both curves are divided by the maximum value for comparison. Vis_FwdFit (blue) fits a Gaussian distribution to the X-ray visibilities, and although the real X-ray profile may deviate from a Gaussian form assumed by Vis_FwdFit (and the CLEAN PSF), the low Vis_FwdFit $\chi^{2}$ values tell us a Gaussian is an adequate approximation for the study.

gradient $\alpha$ has a value of about $+0.2 \operatorname{arcsec} / \mathrm{keV}$, which means the height difference between the peak positions of the minimum and maximum energies of 10 and $25 \mathrm{keV}$ is about $3^{\prime \prime}$.

3. The $\operatorname{DEM}(T)$ derived from modelling should be consistent with the DEM used to fit the spatially integrated RHESSI spectrum (see Fig. 4). From RHESSI spectroscopy, we noted that the OSPEX model DEM functions agree well within the temperature range of $\sim 1.6$ to $3.0 \mathrm{keV}$.

The main results are presented in Fig. 8, which has six panels showing: (1) $n^{2}(z) A(z)$, (2) $T(z)$, (3) X-ray distribution at various photon energies $\epsilon$, (4) The position of the X-ray distribution peak versus energy, (5) X-ray distribution widths (FWHM) versus energy, (6) DEM versus temperature.

We note that RHESSI imaging observations at a given en$\operatorname{ergy} \epsilon$ show that the X-ray profiles along $z$ can be well-described by a Gaussian distribution (i.e. using Vis_FwdFit, even if a Gaussian is not the exact, true form of the profile - see Fig. 6). Hence from Eq. (6), the easiest way to produce a Gaussian profile, is to create a $n^{2}(z) A(z)$ profile that is also Gaussian or close to Gaussian along $z$. Hence taking $n^{2}(z) A(z)\left[\mathrm{cm}^{-4}\right]$ as a Gaussian distribution, we have

$n^{2}(z) A(z)=n_{0}^{2} A_{0} \exp \left(-\frac{\left(z-z_{s}\right)^{2}}{2 \sigma^{2}}\right)$

with centroid $z_{s}$ and $F W H M=2 \sqrt{2 \ln 2} \sigma$. Here, we choose $F W H M=14^{\prime \prime} .5$ and $z_{s}=12^{\prime \prime} .5$, as measured above the solar X-ray limb, and $n_{0}^{2} A_{0}=6 \times 10^{40} \mathrm{~cm}^{-4}$. All these values are found via trial and error, and they are chosen because they produce the main flare observables. For a constant value of $A$, Eq. (14) suggests that the number density would have to fall an order of magnitude or more with increasing $z$, as can be deduced from Panel 1 in Fig. 8. For the chosen $n^{2}(z) A(z)$, the peaks of the X-ray source at a given energy only appear when $\mathrm{d}\left(n^{2} A\right) / \mathrm{d} z$ becomes negative for $z>z_{s}$ (to the right of the black dotted line in Fig. 8), i.e. X-ray emission appears at lower altitudes but the peaks of the X-ray source at a given energy can only appear for $z>z_{\mathrm{s}}$.

The temperature $T(z)$, as function of vertical height $z$, is modelled as a power-law with fixed minimum and maximum temperatures (Fig. 8), so that

$T(z)=0.5+3.2\left(\frac{z-z_{\min }}{z_{\max }-z_{\min }}\right)^{\gamma} \mathrm{keV}$

where $0.5 \mathrm{keV}(\sim 6 \mathrm{MK})$ at $z_{\min }=1^{\prime \prime}$ and $3.7 \mathrm{keV}(\sim 43 \mathrm{MK})$ at $z_{\max }=40^{\prime \prime}$. As discussed in Sect. 4.3, the observations require $T(z)$ increasing with height $z$, so that $\mathrm{d} T / \mathrm{d} z>0$. The temperature gradient in Eq. (15) is changed by varying the power index $\gamma$. Figure 8 shows the variations of $\gamma$ between 0.95 and 1.15 (grey region). This represents a range of $T(z)$ that can best reproduce all the RHESSI observations within uncertainties, while the red curve represents the $T(z)$ distribution with $\gamma \simeq 1.1$ that best fits all the RHESSI observations.

Panel 4 in Fig. 8 shows the resulting X-ray flux against height $z$, for five chosen X-ray energies, chosen to match the average values of the energy ranges used for RHESSI imaging observations. Since $n^{2} A$ was input as a Gaussian, the resulting $I(z)$ at each energy $\epsilon$ are also close to Gaussian, as suggested by the observations. The model X-ray peak positions versus energy give a gradient of $\mathrm{d} z / \mathrm{d} \epsilon \simeq 0.37 \mathrm{arcsec} / \mathrm{keV}$, which is relatively close to the observed $\mathrm{d} z / \mathrm{d} \epsilon=0.24 \mathrm{arcsec} / \mathrm{keV}$. The best-fit X-ray peak positions also appear at a height $z$ of $\sim 20^{\prime \prime}$ (at $10 \mathrm{keV}$ ), as suggested by RHESSI imaging. The average $F W H M \simeq 11^{\prime \prime}$ matches the observed average, and shows that the width decreases with energy as suggested by the images.

In the temperature range between $1.6 \mathrm{keV}$ and $3.0 \mathrm{keV}$, all modelled DEM match well with the DEM models fitted to RHESSI spectrum.

The model emission measure $E M$

$E M=\int_{T} \mathrm{DEM} \mathrm{d} T$ 


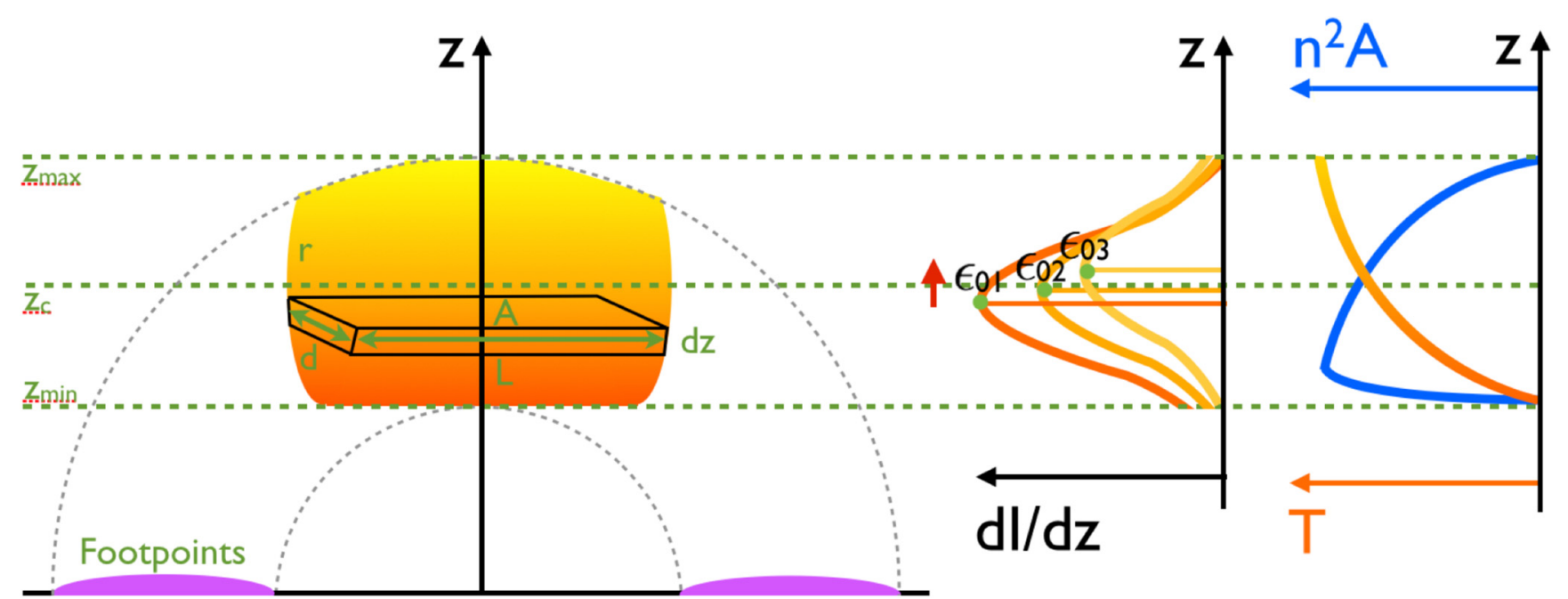

Fig. 7. Left: sketch of the coronal X-ray source geometry, as seen by RHESSI. The lower temperature plasma is located below the higher temperature plasma. A small volume of emitting plasma $\mathrm{d} V=A \mathrm{~d} z=L d \mathrm{~d} z$ varies with altitude $z$. Right: cartoon of the X-ray distribution versus $z$ at energies $\epsilon_{01}<\epsilon_{02}<\epsilon_{03}$ created by varying temperature $T$ and $n^{2} A$ distributions with altitude $z$. The peak X-ray positions at a given energy, as viewed by RHESSI, are related to a $T$ and $n^{2} A$ value at that position.

and the average temperature $\langle T\rangle$

$$
\langle T\rangle=\frac{\int_{T} T \mathrm{DEM} \mathrm{d} T}{\int_{T} \mathrm{DEM} \mathrm{d} T}
$$

are found to be $E M \sim 6.7 \times 10^{49} \mathrm{~cm}^{-3}$ and $\langle T\rangle=1.4 \mathrm{keV}$ $(\sim 16 \mathrm{MK})$.

Over the temperature range between 1.6 and $2.5 \mathrm{keV}$, the model $z$ varies from around $\sim 15^{\prime \prime}$ to $\sim 25^{\prime \prime}$ (see Fig. 8 Panel 2). This leads to a model temperature gradient of $\mathrm{d} T / \mathrm{d} z \sim 0.08 \mathrm{keV} / \operatorname{arcsec}$. From the model, it is suggested that the form of the DEM at lower temperatures below $1.6 \mathrm{keV}$ is closer to both the Gaussian and power-law exponential OSPEX models, which could not be confidently determined by spectroscopy alone.

In Fig. 9 (right most panel), we also show that such a trend is common at different times during the studied flare, with the gradient $\mathrm{d} R / \mathrm{d} \epsilon$ varying at different times. However, changes in time are beyond the scope of this paper. The temporal changes are the subject of ongoing studies. In Fig. 9 (middle and left most panels), we also show that the coronal X-ray source altitude increases with energy for two other flares studied at a single time range close to the peak flux of each flare.

\section{Summary}

A detailed analysis of solar flare SOL2013-05-13T02:12, during a single time interval, was performed using RHESSI imaging and spectroscopy observations. We investigated quantitatively the increase in altitude of the flare coronal X-ray source with energy at a single time interval during the flare impulsive phase, a trend that has been noted previously (e.g. Jeffrey \& Kontar 2013).

The RHESSI imaging analysis shows that the peaks of X-ray emission in the coronal X-ray source are located close together over a small distance of $3^{\prime \prime}$ between the X-ray energies of 10 and $25 \mathrm{keV}$. The increase in source height with photon energy can be well-fitted by a linear function with a gradient of $\mathrm{d} z / \mathrm{d} \epsilon \simeq 0.2 \mathrm{arcsec} / \mathrm{keV}$. RHESSI spectroscopy showed that a number of different thermal models (both isothermal and multithermal) can fit the spatially integrated X-ray spectrum adequately well. At the same time, the analysis of the flare data shows that the emitting flare plasma in the corona cannot be isothermal, as an isothermal plasma is unable to account for the observed changes in altitude with X-ray energy, as shown by RHESSI imaging. This is an important result for deriving the properties of hot X-ray emitting plasma in the flaring solar corona, which is often performed with an isothermal model.

Further, our study shows how RHESSI imaging can be used to constrain the properties of flaring plasma beyond what is possible using X-ray spectroscopy alone. Coronal X-ray emission in the range of 10 to $25 \mathrm{keV}$ can be well explained using model distributions in temperature, number density and area, that vary with altitude. Modelling can adequately explain all the main observations: radial position (gradient $\mathrm{d} z / \mathrm{d} \epsilon$ and approximate height above the X-ray limb), vertical source size, and differential emission measure. For our model, the temperature and $n^{2} A$ gradients $\left(\mathrm{d} T / \mathrm{d} z\right.$ and $\left.\mathrm{d} n^{2} A / \mathrm{d} z\right)$ must have opposite signs and the $n^{2} A$ distribution must decrease over a portion of the region with increasing altitude $z$, in order to produce the RHESSI imaging results. The modelling suggested that $\mathrm{d} T / \mathrm{d} z$ between 1.6 and $2.5 \mathrm{keV}$ should be $\sim 0.08 \mathrm{keV} / \operatorname{arcsec}$. Since this gradient occurs over most of the observable region $\left(\sim 15^{\prime \prime}\right)$, it may be used in future analysis to distinguish between different cooling and heating processes occurring within the flaring coronal X-ray source. A constant area $A$ suggests that the number density should decrease over an order of magnitude with altitude, although $A$ might also vary with $z$, as suggested by observations of $\mathrm{X}$-ray source length and width variations. Again, such a prediction should help constrain the processes occurring in the coronal region of solar flares.

We have analysed a number of other limb flares (see Fig. 9 for some examples) with bright coronal X-ray sources, and they all show the source altitude increasing with increasing X-ray energy. This suggests that the observations presented here are common for a majority of coronal sources, and hence vertical temperature and density gradients with rather broad multi-thermal $\operatorname{DEM}(T)$ s are common. 
N. L. S. Jeffrey et al.: The temperature and density structures of coronal X-ray sources
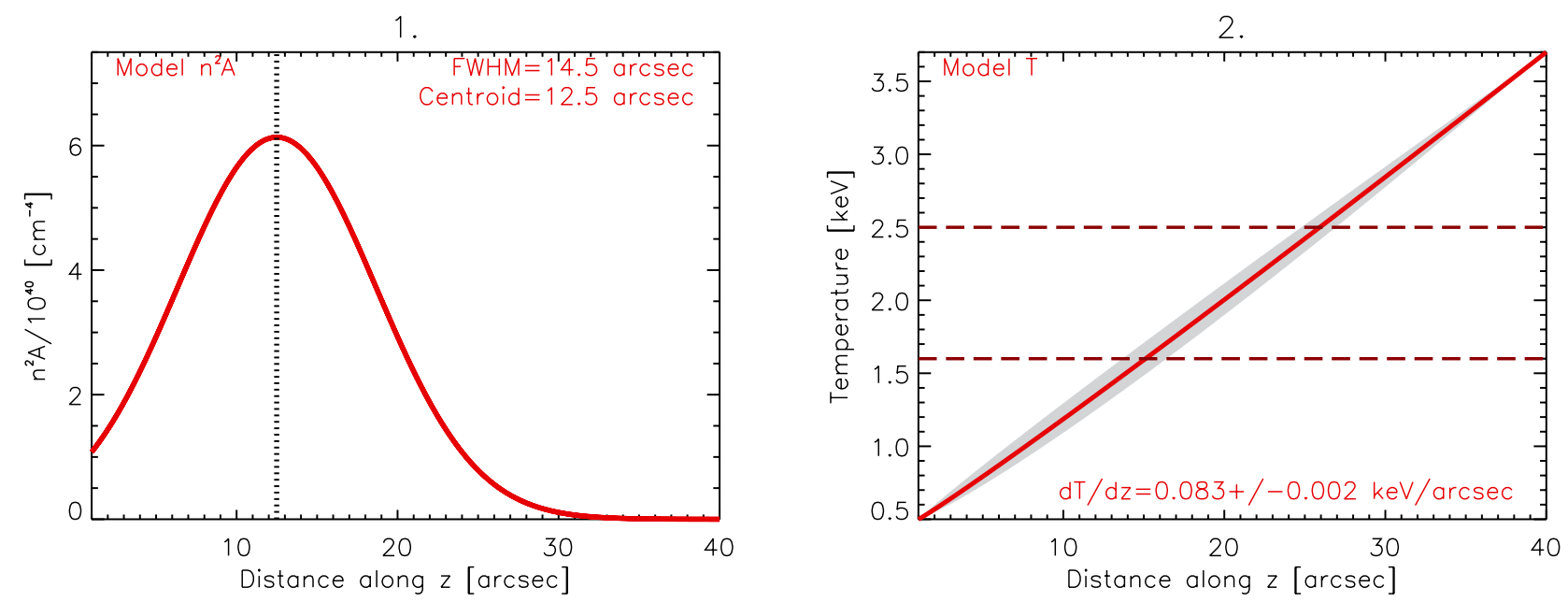

3.
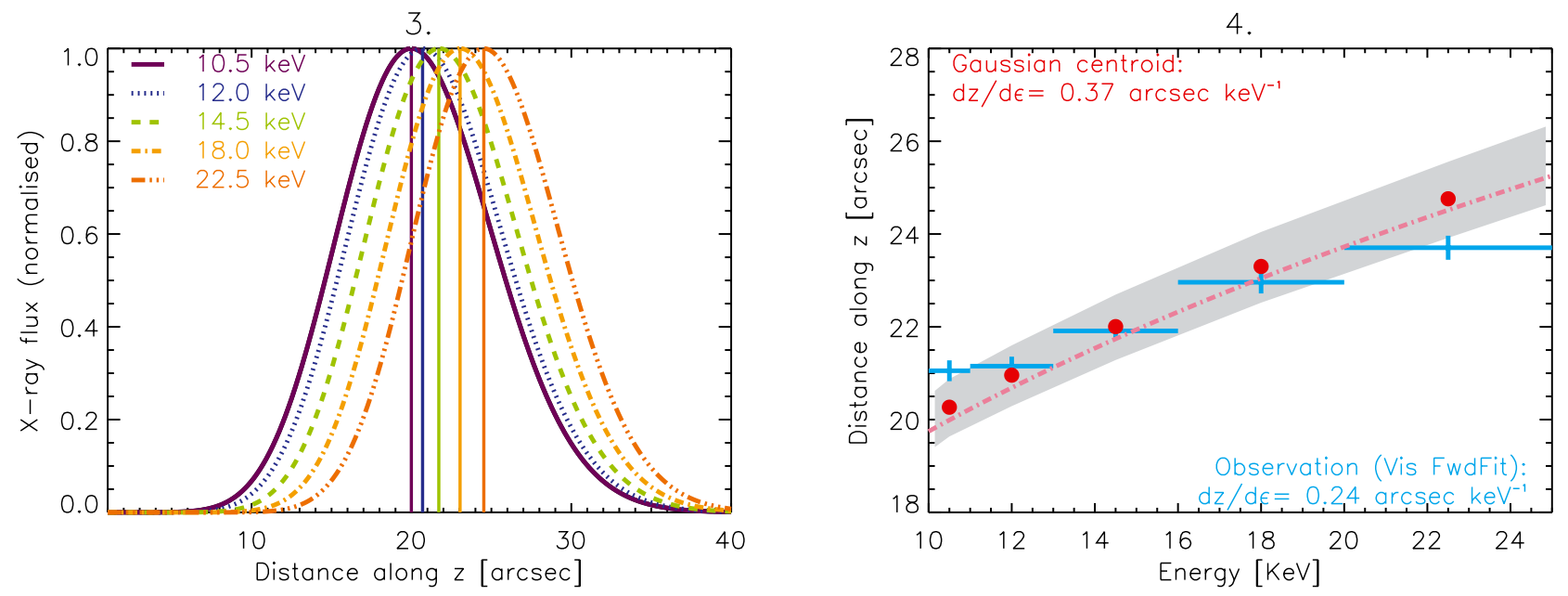

5.

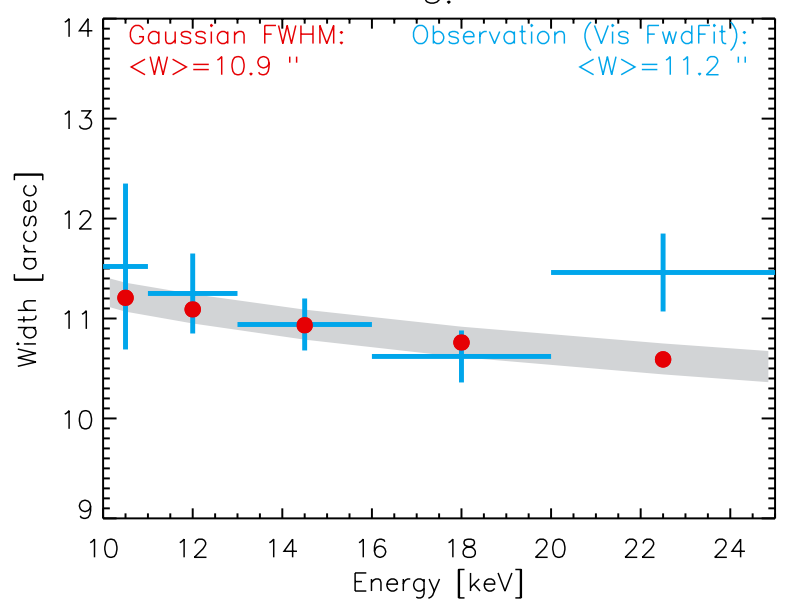

6.

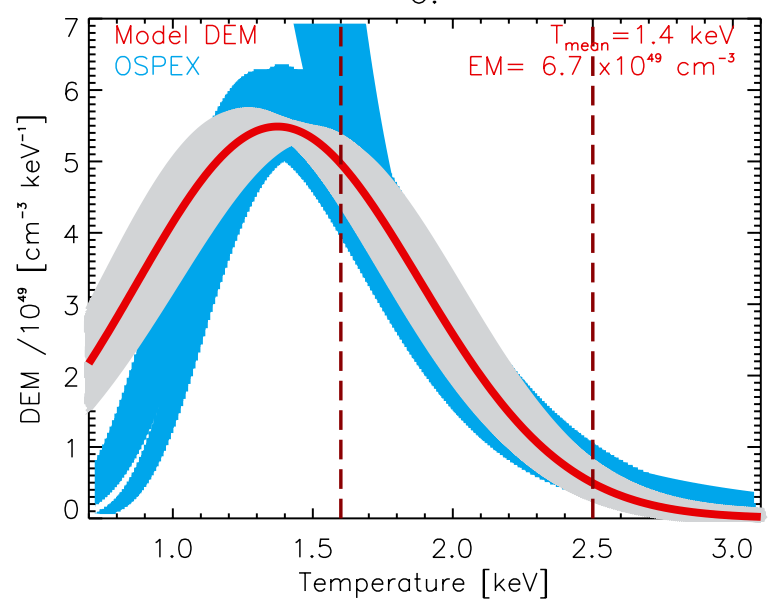

Fig. 8. Temperature and density-altitude distribution model (see Sect. 5 for details) with 6 different panels. In all panels, the best model result is represented by the red line and points. $1: n^{2} A$ vs. height $z$ given by Eq. (14). 2: Temperature $T$ vs. $z$ given by Eq. (15). 3: X-ray distribution at various photon energies $\epsilon$ vs. altitude. The vertical lines indicate the peak locations. 4: The model X-ray distribution peak position vs. energy (red dots) and Eq. (9) using the model $T(z)$ and $n^{2}(z) A(z)$ (pink dashed-dotted line). 5: X-ray distribution widths (FWHM) vs. energy. 6: Differential emission measure, DEM $(T)$. Panels 4-6 show both the results of the RHESSI data analysis (in blue) and the model predictions (in red). The grey areas show the range of each parameter that can adequately match the measured values using the range of modelled $T(z)$ shown in Panel 2.

One notable limitation of RHESSI is the insensitivity to plasma at temperatures below $\sim 1 \mathrm{keV}$. Different imaging instruments should be used to extend the range of temperatures (e.g. Battaglia \& Kontar 2013). We plan to better constrain the electron number density and temperature distributions of a flaring coronal region, supplementing existing RHESSI data with EUV imaging and spectroscopy data. This will allow us to extend our imaging approach to temperatures down to a few MK.

In conclusion, we have demonstrated how imaging should be used to improve spectral analysis, allowing the shape of the 

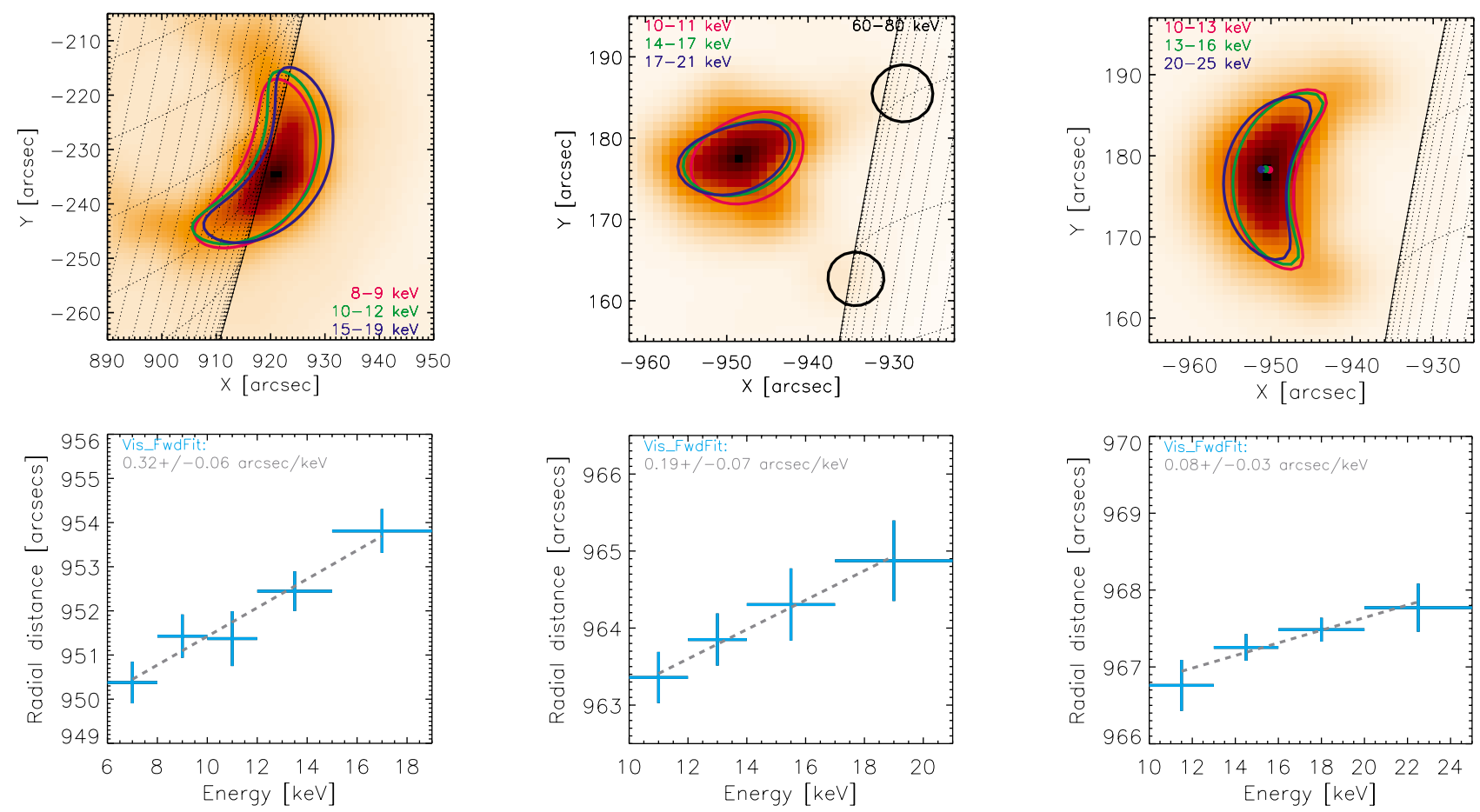

Fig. 9. Coronal X-ray sources in different energy bands, for two additional flares: SOL2005-08-23T14:32 from 14:30 to 14:33 UT (left) and SOL2013-05-13T16:05 from 16:02 to 16:04 UT (middle). Both flares are imaged during the time of peak X-ray flux, and the source altitude increases with X-ray photon energy in these two flares. This trend also occurs for the analysed flare SOL2013-05-13T02:12 at different times $($ right). Here the imaging results are shown for the time of 02:04 to 02:06 UT during the rise phase of the flare, showing that the gradient $\mathrm{d} R / \mathrm{d} \epsilon$ varies with time.

$\operatorname{DEM}(T)$ to be better deduced, with X-ray imaging providing a relatively simple guidance to the $n^{2}(z) A(z)$ and temperature $T(z)$ profiles within a flaring coronal X-ray source.

Acknowledgements. N.L.S.J. was funded by a STFC STEP award. E.P.K. gratefully acknowledges the financial support by the STFC Consolidated Grant.

\section{References}

Aschwanden, M. J., Brown, J. C., \& Kontar, E. P. 2002, Sol. Phys., 210, 383 Battaglia, M., \& Kontar, E. P. 2013, ApJ, 779, 107

Dere, K. P., Landi, E., Mason, H. E., Monsignori Fossi, B. C., \& Young, P. R. 1997, A\&AS, 125, 149

Forbes, T. G., \& Acton, L. W. 1996, ApJ, 459, 330

Gallagher, P. T., Dennis, B. R., Krucker, S., Schwartz, R. A., \& Tolbert, A. K. 2002, Sol. Phys., 210, 341

Gosain, S. 2012, ApJ, 749, 85

Guo, J., Emslie, A. G., Massone, A. M., \& Piana, M. 2012, ApJ, 755, 32

Guo, J., Emslie, A. G., \& Piana, M. 2013, ApJ, 766, 28

Högbom, J. A. 1974, A\&AS, 15, 417

Holman, G. D., Aschwanden, M. J., Aurass, H., et al. 2011, Space Sci. Rev., 159, 107

Hurford, G. J., Schmahl, E. J., Schwartz, R. A., et al. 2002, Sol. Phys., 210, 61 Ireland, J., Tolbert, A. K., Schwartz, R. A., Holman, G. D., \& Dennis, B. R. 2013, ApJ, 769, 89

Jeffrey, N. L. S., \& Kontar, E. P. 2013, ApJ, 766, 75

Jeffrey, N. L. S., Kontar, E. P., Bian, N. H., \& Emslie, A. G. 2014, ApJ, 787, 86
Joshi, B., Veronig, A., Cho, K.-S., et al. 2009, ApJ, 706, 1438

Kontar, E. P., Hannah, I. G., \& MacKinnon, A. L. 2008, A\&A, 489, L57

Kontar, E. P., Hannah, I. G., Jeffrey, N. L. S., \& Battaglia, M. 2010, ApJ, 717, 250

Kontar, E. P., Brown, J. C., Emslie, A. G., et al. 2011a, Space Sci. Rev., 159, 301 Kontar, E. P., Hannah, I. G., \& Bian, N. H. 2011b, ApJ, 730, L22

Landi, E., Young, P. R., Dere, K. P., Del Zanna, G., \& Mason, H. E. 2013, ApJ, 763,86

Lemen, J. R., Title, A. M., Akin, D. J., et al. 2012, Sol. Phys., 275, 17

Lin, R. P., Dennis, B. R., Hurford, G. J., et al. 2002, Sol. Phys., 210, 3

Liu, R., Wang, H., \& Alexander, D. 2009, ApJ, 696, 121

Liu, W., Chen, Q., \& Petrosian, V. 2013, ApJ, 767, 168

Phillips, K. J. H., Chifor, C., \& Dennis, B. R. 2006, ApJ, 647, 1480

Prato, M., Piana, M., Brown, J. C., et al. 2006, Sol. Phys., 237, 61

Reznikova, V. E., Melnikov, V. F., Ji, H., \& Shibasaki, K. 2010, ApJ, 724, 171

Schmahl, E. J., Pernak, R. L., Hurford, G. J., Lee, J., \& Bong, S. 2007, Sol. Phys., 240,241

Schwartz, R. A., Csillaghy, A., Tolbert, A. K., et al. 2002, Sol. Phys., 210, 165

Sui, L., \& Holman, G. D. 2003, ApJ, 596, L251

Sui, L., Holman, G. D., \& Dennis, B. R. 2004, ApJ, 612, 546

Švestka, Z. 1996, Sol. Phys., 169, 403

Svestka, Z. F., Fontenla, J. M., Machado, M. E., Martin, S. F., \& Neidig, D. F. 1987, Sol. Phys., 108, 237

Tandberg-Hanssen, E., \& Emslie, A. G. 1988, The physics of solar flares (Cambridge University Press)

Tsuneta, S., Hara, H., Shimizu, T., et al. 1992, PASJ, 44, L63

Veronig, A. M., \& Brown, J. C. 2004, ApJ, 603, L117

Veronig, A. M., Karlický, M., Vršnak, B., et al. 2006, A\&A, 446, 675

Xu, Y., Emslie, A. G., \& Hurford, G. J. 2008, ApJ, 673, 576 